\title{
DISTRIBUTED WEB-BASED EXPERT SYSTEM FOR LAUNCH OPERATIONS
}

\author{
Jorge E. Bardina \\ Intelligent Systems Division \\ NASA Ames Research Center \\ Moffett Field, CA 94035, U.S.A.
}

\author{
Rajkumar Thirumalainambi \\ SAIC@ Intelligent Systems Division \\ NASA Ames Research Center \\ Moffett Field, CA 94035, U.S.A.
}

\begin{abstract}
The simulation and modeling of launch operations is based on a representation of the organization of the operations suitable to experiment of the physical, procedural, software, hardware and psychological aspects of space flight operations. The virtual test bed consists of a weather expert system to advice on the effect of weather to the launch operations. It also simulates toxic gas dispersion model, and the risk impact on human health. Since all modeling and simulation is based on the internet, it could reduce the cost of operations of launch and range safety by conducting extensive research before a particular launch. Each model has an independent decision making module to derive the best decision for launch.
\end{abstract}

\section{INTRODUCTION}

As NASA plans to launch humans for Mars Mission with an advanced Crew Exploration Vehicle based on nuclear propulsion technology, the launch operations are rapidly becoming very complex in control and communications. To overcome this complex launch operations, increasing number of experts are required for precise launch operations. The experts in various domains are distributed over the continental US and it is less likely that everyone can be present near launch pad or operational centers. Additionally, if multiple NASA centers require the expertise at the same time, the information may not be available in realtime to provide the required assistance. Given these facts it is becoming increasingly important to rely on technology to provide such expertise.

One feasible solution for providing distributed expert systems is the World Wide Web (WWW). The WWW permits the expert's knowledge to be provided at such distributed locations. There are many factors that can affect the decision to use the WWW as a delivery mechanism for expert knowledge. The crucial factors in implementing at NASA are security and fast communication within NASA.

\section{EXPERT SYSTEMS}

NASA has developed a set of contingency rules for launch commit criteria. A distributed expert system for launch operations can operate concurrently to derive ultimate "Go/No-Go" decision within the scenario of a launch or landing of a space vehicle (Bardina and Thirumalainambi, 2003).

Here, we have implemented three different expert systems, which derive decisions based on launch contingency rules, the weather expert system, the toxic-gas exposure expert systems, and the human-health risk assessment expert system. A dedicated web server with data management system and physical models supports each expert system.

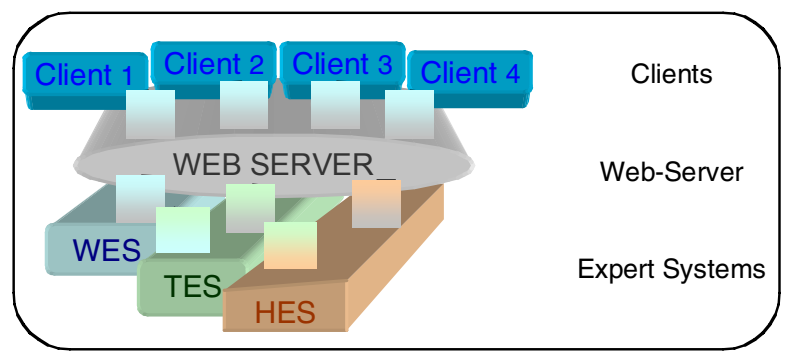

Figure 1: Web Server Expert System

Each expert system consists of an inference engine based on backward chaining process, knowledge base based on specific domains (for example, weather, toxic gas dispersion and human health risk assessment contingency rules for launch commit criteria) and web based graphical user interface for user interaction. Each expert system has specific type of rules which are derived from contingency rules. The interoperability among expert systems is based on internet protocol. Although one expert system (originator) can get the information about capabilities of another expert system (recipient) through the internet, it is important to identify the information available to improve the decision-making capability of the originator. A method to find out the difference arising in the context of the corre- 


\section{Bardina and Thirumalainambi}

spondence between inference primitive of an originator and those of a recipient is presented here as follows:

- Make a set of correspondence in which inference primitives are the same between an originator and a recipient.

- By taking a look at the context (pre-inferenceprimitive, post-inference primitive, input, output and reference knowledge) of the inference primitive in the correspondence, the correspondence value is computed. The more similar the context, the larger the value.

- The correspondence value is propagated to preand post- inference primitives.

- After completing propagation over all inference primitives in the correspondence, the difference arising in the context of the correspondence with large values can be used as a reply message to modify the originator's inference engine.

The extreme complexity of the launch and range operations also require cooperation among the multiple advisory expert systems; in this paper, crucial expert systems: weather expert system, toxic-gas expert system, and human-health risk assessment expert system) and their interoperability are included.

The expert systems are supported by real-time and model data to make an optimized decision-making. The uncertainty involved in decision-making process is the reflectance of the physical models rather than expert system by itself. The U.S.A. Air force authority depending on the criticality and implications of the decision can waive conflicting decisions of launch operations. If there is a lightning strike before 15 minutes of launch, the launch will be on hold, until all the clouds and suitable weather permits the launch. This distributed expert system acts as an advisory system and can be transformed into autonomous decision support system by implementing suitable intelligent agents among the distributed expert system.

\subsection{Weather Expert System}

The Weather expert system (WES) is a crucial system for launch decision-making (Rajkumar and Bardina 2003).

The weather rules characterize certain aspects of the environment related to the launching or landing site, the time of the day or night, the pad or runway conditions, the mission durations, the runway equipment and landing type. Expert system rules are derived from weather contingency rules, which were developed over several years by NASA. Backward chaining, a goal-directed inference method is adopted, because a particular consequence or goal clause is evaluated first, and then chained backward through the rules. Once a rule is satisfied or true, then that particular rule is fired and the decision is expressed. The expert sys- tem is continuously verifying the rules against the past one-hour weather conditions and the decisions are made.

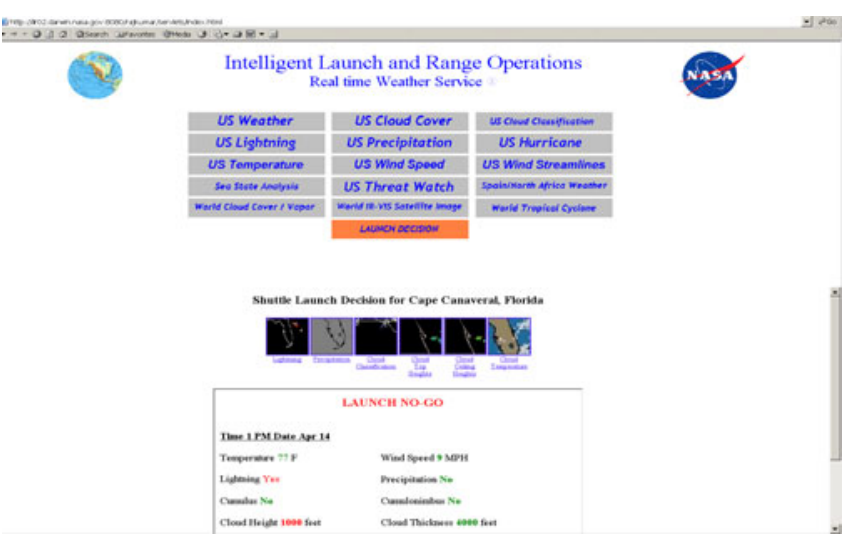

Figure 2: WES Graphical User Interface

The normal procedure of operations requires a formal pre-launch weather briefing held on Launch minus 1 day, which is a specific weather briefing for all areas of Space Shuttle launch operations. WES acquires data for continental US and World weather. It can be applicable to any launch site in the US by providing the Shuttle launch site rules. The user can define any number of rules and the expert system is very flexible and robust.

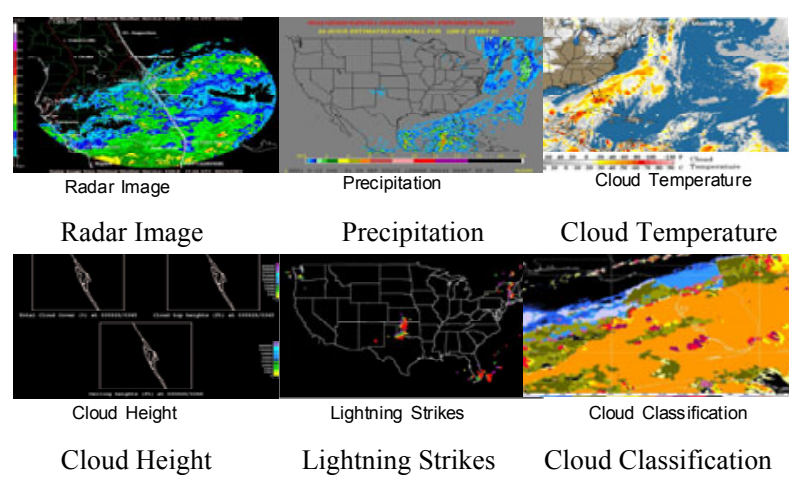

Figure 3: Weather Data from Satellites and Radar

\subsection{Toxic-Gas Expert System}

The burning of rocket engines during the first few seconds to prior and immediately following vehicle launches results in the formation of a large cloud of hot, buoyant exhaust products near the ground level which subsequently rises and entrains ambient air until the temperature and density of the cloud reach an approximate equilibrium with ambient conditions. This cloud is referred to ground-cloud. The rocket engines also leave an exhaust trial from normal launches which extend throughout the troposphere and beyond. The toxic gas dispersion model calculates peak con- 


\section{Bardina and Thirumalainambi}

centration and deposition (resulting from gravitational and precipitation scavenging) downwind from normal launches.

The algorithm models of toxic-gas dispersion are shown in Figure 4 (Bardina and Thirumalainambi 2004a and 2004b). The required meteorological inputs for the gas dispersion model are vertical profiles of wind direction, wind speed, air temperature, pressure and dew point or relative humidity between the earth's surface and $3000 \mathrm{~m}$ (Baskett and Pace 1995). This information is obtained during launch support activities from Rawinsonde measurements routinely made at scheduled times throughout the pre-launch countdown and after the launch has occurred (Boyd 1985, and FAA 1999). The wind system is a series of $30 \mathrm{~m}$ towers located throughout launch site to measure wind direction, wind speed, turbulence and air temperature. The type of launch vehicle and launch modes (normal, deflagration and conflagration) can also be selected via web interface in Toxic gas dispersion model web server. Once required inputs for models have been provided, peak centerline concentration (ppm) along the trajectory is computed at $1 \mathrm{~km}$ intervals along down range from launch pad.

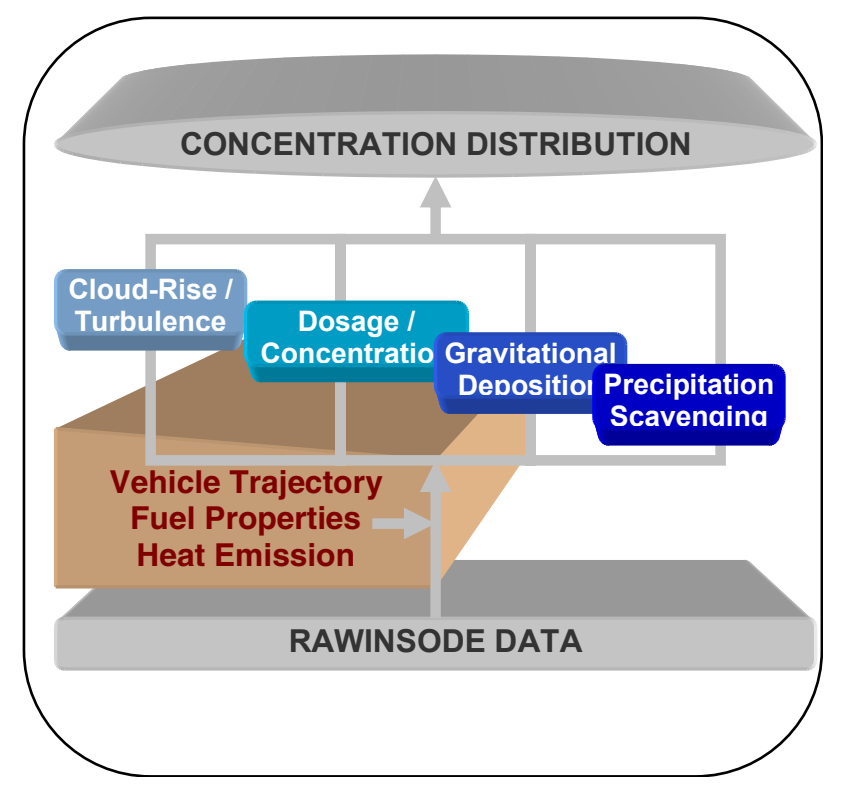

Figure 4: Toxic-Gas Dispersion Algorithm Models

The fuel expenditure rates for normal launches are obtained by averaging the fuel expenditure rates for the engines over the approximate period from lift-off until the vehicle is about $3000 \mathrm{~m}$ above the surface. The effective fuel heat contents, which are used in calculating buoyant cloud rise for normal launches and plume rise for launch failures, include the effects of heat produced by after burning as well as heat losses due to radiation. The toxic dispersion model is based on Gaussian approach which is a practical diffusion modeling tool. It is mathematically sim- ple and flexible. The basic assumption in the model is the exhaust material is assumed to be uniformly distributed in the vertical and to have a bi-variate Gaussian distribution in the plane of the horizon at the point of cloud stabilization (Beychok 1995). The dosage and concentration are written in a rectangular co-ordinate system with the origin at the launch pad. One axis is directed along the mean wind direction in a given altitude layer and the other axis is directed crosswind or perpendicular to the mean wind direction. A backward chaining inference engine is applied for the expert system and it is presently focusing $\mathrm{HCl}$ chemical alone. The output of the toxic gas dispersion model forms as an input to the toxic gas expert system. The rules for the toxic gas expert system (TES) are based on downrange, peak chemical concentration and type of launch.

\subsection{Human Health Risk Assessment Expert System}

During a launch of a rocket under prevailing weather conditions, commanders at US Air Force (USAF) station evaluate the possibility of wind blown toxic emissions might reach civilian and military personnel near by area (Rajkumar and Bardina 2004). The Air Force uses a model called "LATRA" which is based on Monte Carlo simulation with limited amount of data for toxic response functions to humans (Bennett and McDonald 1999, and Hudson et al. 1999). In our model, we focused mainly HCL, HNO3 and Nitrogen dioxide (NO2), which are non-carcinogenic chemicals as per United States Environmental Protection Agency (US EPA) classification. Without specific incidence data (e.g. mortality, acute illness etc) on humans or animals, it is difficult to endorse a particular exposure response model to predict incidences. For the hazard quotient (HQ) model, estimates of number of people at risk would be based on the number of people with exposure above a reference exposure level that is unlikely to cause adverse health effects (Yassi 1998, NASA 1988, and NRC 1998 and 2000). The ratio of exposure concentrations to reference exposure levels might be useful. The hazard quotient model is simply a comparison of the estimated exposure concentration (EEC) to a NOEL or other reference toxicity value (RTV). When the ratio of the EEC to the RTV value is less than 1, effects are considered unlikely. When the quotient is greater than 1 , some effects might occur in some individuals. Interpretation of the ratio of the two values depends on the uncertainty associated with each. As the value of the EEC/RTV increases, both the severity and incidence of effect are likely to increase, but the ratio is not used to predict incidence or severity. In a risk assessment, the magnitude of EEC/RTV helps to estimate the severity of the effects of exposure. An additional advantage of the hazard quotient model is that it allows estimation of the number of people at risk of additive effects from simultaneous exposure to two or more substances that is not possible in a traditional risk assessment model. The 


\section{Bardina and Thirumalainambi}

risk to the exposed population is calculated by multiplying the individual risk and the number in exposed population (this should take into consideration age and other susceptibility factors and population activities etc). Based on HQ model's threshold, a decision is derived how launch operations affects nearby residents.

The following Table 1 shows the US Air Force Tier exposure limits for Rocket emission toxicants.

Table 1. U.S.A. Air Force Tier Exposure Limits.

\begin{tabular}{|c|c|}
\hline Chemical Species & Tier 1 (outer) PPM \\
\hline $\mathrm{HCl}$ & 10 , ceiling $2,1-\mathrm{hr}$ TWA \\
\hline $\mathrm{N}_{2} \mathrm{O}_{4}\left(\right.$ as $\left.\mathrm{NO}_{2}\right)$ & $0.2,1-\mathrm{hr}$ TWA, 2 , ceiling \\
\hline $\mathrm{HNO}_{3}$ & 0.3 , ceiling $0.025,1-\mathrm{hr}$ TWA \\
\hline
\end{tabular}

\begin{tabular}{|c|c|}
\hline Chemical Species & Tier 2 (middle) PPM \\
\hline $\mathrm{HCl}$ & 10 , ceiling \\
\hline $\mathrm{N}_{2} \mathrm{O}_{4}\left(\right.$ as $\left.\mathrm{NO}_{2}\right)$ & $2,1-\mathrm{hr}$ TWA, 4, ceiling \\
\hline $\mathrm{HNO}_{3}$ & $2.5,1-\mathrm{hr}$ TWA, 4, ceiling \\
\hline
\end{tabular}

\begin{tabular}{|c|c|}
\hline Chemical Species & Tier 3 (inner) PPM \\
\hline $\mathrm{HCl}$ & 50 , ceiling \\
\hline $\mathrm{N}_{2} \mathrm{O}_{4}\left(\right.$ as $\left.\mathrm{NO}_{2}\right)$ & 20,30 -min TWA \\
\hline $\mathrm{HNO}_{3}$ & 25,30 -min TWA \\
\hline
\end{tabular}

The US Air Force adopts a three-tiered concept to delineate acceptable exposure concentrations and durations for the public (tier 1), government and contractor personnel on the ranges (tier 2), and operational personnel directly involved with the launch (tier 3). The Air Force defines the tier 1 exposure limit (the outermost tier) as the airborne exposure concentration that poses no hazard to the general population but might affect certain sensitive individuals (e.g., individuals with asthma or emphysema and people with certain other lung diseases; US Air Force 1997). If tier 1 concentrations are exceeded beyond the base, the Air Force notifies public officials. The Air Force defines the tier 2 exposure limit (the middle tier) as the airborne exposure concentration that might cause short-term symptoms that most individuals could endure without experiencing or developing irreversible or other serious health effects or symptoms that could impair their ability to take protective action. For personnel in areas with a tier 2 concentration, the Air Force recommends seeking shelter and evacuation. If tier 2 concentrations are predicted to overlay unprotected population centers on or off-base, the Air Force wing safety office recommends a launch hold to the wing commander. The Air Force defines the tier 3 exposure limit (the innermost tier surrounding the launch pad) as an airborne exposure concentration that can be immediately dangerous to life and health (IDLH). Tier 3 exposure concentrations are based on the NIOSH IDLH (1994) values. Areas within tier 3 concentrations warrant immediate isolation and evacuation actions to prevent exposure.
The centerline peak concentrations of each one of the chemical species are compared against various US Air force tier level concentrations and decisions are suggested based on acceptable exposure concentrations. These decisions are based on threshold values. All decisions are unified together to derive to a final decision for launch operations.

The decision-making process for launch commit criteria can be supported by an intelligent system composed of autonomous agents based on individual expert systems to support present and future space missions (Watson 1997, and Bigus and Bigus 2001).

\section{DISTRIBUTED WEB-BASED SYSTEM}

The interoperability among expert systems is based on Internet protocol. Apart from the inference process of expert systems, data is also shared among the expert systems; Tomcat web servers distributing the information to the clients through Internet browsers enable this horizontal cooperation among expert systems.

\subsection{Launch Decision Support System}

Before deriving launch decisions, the near real-time weather data has to be collected near the launch pad and the planned trajectory of the vehicle, so that weather forecasting models can predict weather during the launch window. The gas dispersion model predicts the toxic gas dispersion in the region of interest. Once the prediction models compute the predicted weather and toxic gas concentration, it can be directed to the corresponding expert system to derive the launch status based on weather, toxicgas dispersion and human health risk. Figure 5 shows the graphical user interface and indicates the inputs that the user has to provide to compute the mean concentration over a region of interest. The launch decision support system with respect to toxic gas dispersion and human health risk assessment can be both invoked together as shown in Figure 5.

Figure 6 shows the rules explaining the reasoning for a particular decision and the rules satisfied for a specific condition. The rules are hyperlinked, as it is shown in Figure 7. This rule has 4 antecedent variables and one consequent variable. Depends on the type of chemical species and type of launch, there are multiple rules to satisfy the range conditions. 


\section{Bardina and Thirumalainambi}

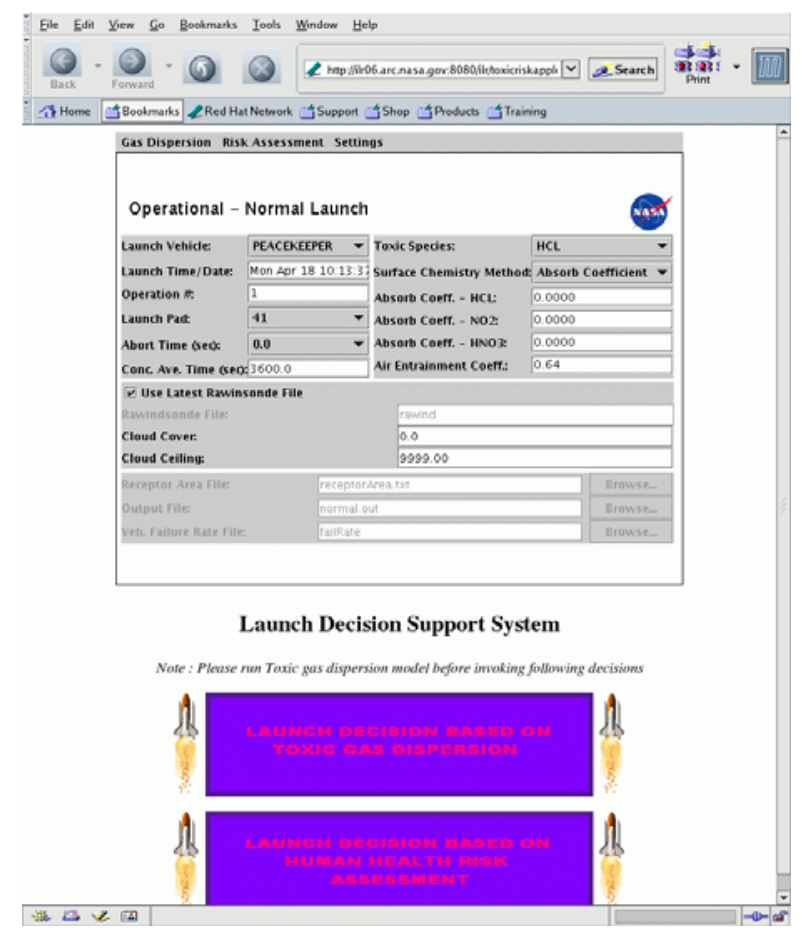

Figure 5: Launch Decision Support System

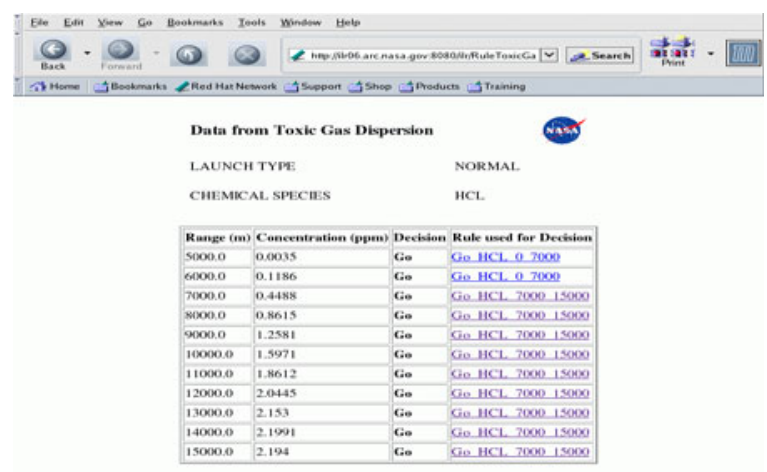

Launch Decision based on Toxic Gas Dispersion : GO

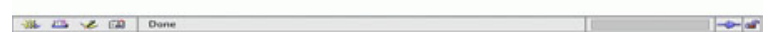

Figure 6: Rules for Launch Decision Support System

$$
\begin{aligned}
& \text { Rule: Go_HCL_7000_15000 } \\
& \text { IF chemical }=\text { HCL AND } \\
& \text { launchtype }=\text { NORMAL AND } \\
& \text { concentration }<\mathbf{1 6} \text { PPM AND } \\
& \text { 6999 } \mathbf{M}<\text { range }<\mathbf{1 5 0 0 1} \text { M THEN } \\
& \text { THEN launch }=\text { GO }
\end{aligned}
$$

Figure 7: Rule used in the Decision-Making Process

\subsection{Human-Health Risk Assessment Support System}

The toxic gas dispersion model computes chemical concentration with respect to down range. These results form as inputs to human health risk assessment models. In the launch decision support system, the Hazard quotient index method and the U.S.A. Air Force method are adopted to derive human health risk assessment. Both methods produce very conservative estimates of assessing a human health risk for a specific chemical species.

The human health risk decision is based on U.S.A. Air force exposure limits, as shown in Figure 8. Java-based software "openmap" is used to display the human health risk over geographical information system (GIS). For displaying purposes, the user can set priority of layers.

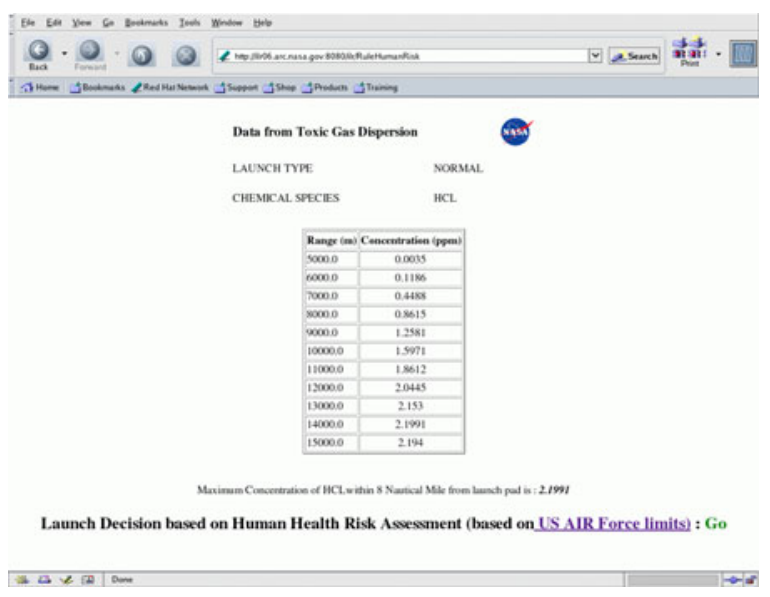

Figure 8: Launch Decision based on the U.S.A. Air Force Exposure Limits

In Figure 9, the left hand side arrows help the user to arrange different layers. The user can turn on and off the layer by turning on and off the bulb. The risk contour based on Hazard Quotient index method for hydrogen chloride is computed and displayed in Figure 10. If hazard index value exceeds 1, there is a significant impact on human health risk. All the models and expert systems are distributed by web server and can be accessed through Internet around the world. 


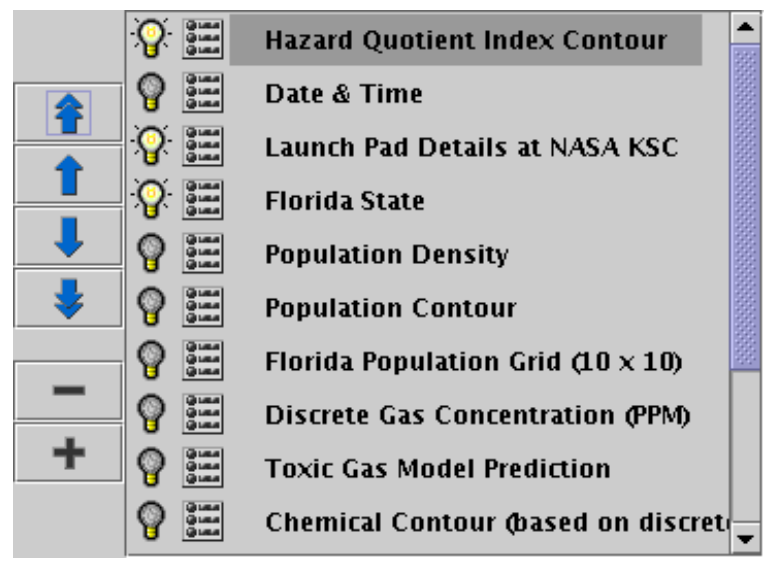

Figure 9: Multiple Layer Rendering using "openmap" Software

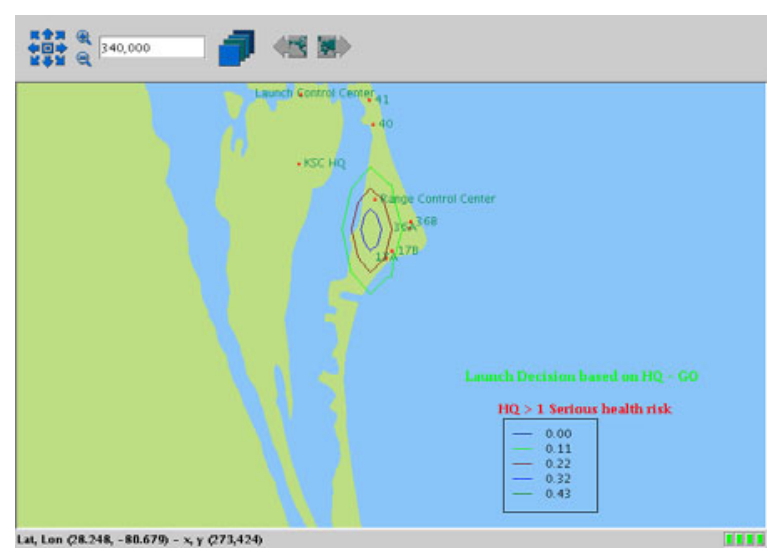

Figure 10: Human Health Risk Assessment based on Hazard Quotient Index

\section{CONCLUSIONS}

Dedicated web servers serve the complex launch and range safety models. Furthermore we are enhancing support for ground operations by adding dedicated web servers. The virtual test bed simulation combines physical model and decision models written in different languages to a unique virtual test bed to visualize complex operations. Further research is planned to develop intelligent agents for each simulation and a decision support system to fully automate space launch initiatives. Since we use Java, it is compatible with any platform and operating system. Each class file in Java can be converted to an applet, Servlet, an application or a library in jar file. The virtual test bed technology enables an entire suite of applications and models for launch and range safety operations.

\section{ACKNOWLEDGMENT}

The authors would like to thank Dr. Michael Shafto and Dr. Butler Hine for their support from NASA's Exploration Systems Enterprise.

\section{REFERENCES}

Bardina, J. and R. Thirumalainambi, 2004a. "Web-Based Toxic Gas Dispersion Model for Shuttle Launch Operations". In Proceedings of the Modeling, Simulation, and Calibration of Space-Based Systems II Conference, (Orlando, FL). SPIE, Orlando, FL, 5420, 136144.

Bardina. J. and R. Thirumalainambi, 2004b. "Modeling and Simulation of Shuttle Launch and Range Operations". In 18th European Simulation Multiconference. ESM, Magdeburg, Germany.

Bardina, J. and R. Thirumalainambi, 2003, "Intelligent Launch and Range Virtual Test Bed (ILR-VTB)". In Proceedings of SPIE Enabling Technologies for Simulation Science VII, (Orlando, FL, March 31). SPIE, Orlando, FL, 5091, 141-148.

Baskett, R and J. Pace. 1995. ARAC Dispersion modeling for January-March 1995 Vandenberg AFB Launches Lawrence Livermore National Laboratory. USA.

Bennett, R. R. and McDonald, A. J. 1999. "Local environmental and toxicity issues for rocket launching and testing". JANNAF $28^{\text {th }}$ propellant development and characterization subcommittee and $17^{\text {th }}$ safety and environmental protection subcommittee joint meeting. 153-173., USA.

Beychok, R. M. 1995. Fundamentals of stack gas dispersion. USA

Bigus, J. and Bigus, J. 2001. Constructing Intelligent agents using Java. John Wiley and Inc. New York. USA.

Boyd, B.F. 1985. "Operational use of REEDM". Weather Squadron $2^{\text {nd }}$ Patrick Air Force Base, Florida. USA.

FAA. 1999. $45^{\text {th }}$ Space wing/Patrick air force base launch site safety assessment. Prepared under contract no. DTFA01-95-D-03009. Licensing and Safety division, Washington D.C. USA.

Hudson, J.M; A.M. See; and L. L. Philipson. 1999. "Launch Area Toxic Risk Analysis (LATRA): Risk management computer Program". JANNAF $28^{\text {th }}$ propellant development and characterization subcommittee and $17^{\text {th }}$ safety and environmental protection subcommittee joint meeting, 183-191., USA.

NASA. 1988. Range safety 1102. Mission Directorate. Flight analysis branch. USA.

NRC. 1998. Assessment of Exposure-Response functions for Rocket-Emission Toxicants. National Academy Press. Washington D.C. USA. 
NRC. 2000. Streamlining Space Launch Range safety. National Academy Press. Washington D.C. USA.

Rajkumar, T. and J. Bardina. 2004. "Human Health Risk Assessment Simulations in a Distributed Environment for Shuttle Launch". In Proceedings of the Modeling, Simulation, and Calibration of Space-Based Systems II Conference, (Orlando, FL). SPIE, Orlando, FL, 5420, 126-135.

Rajkumar, T. and J. Bardina. 2003. "Web-based Weather Expert System (WES) for Space Shuttle Launch". In Proc. IEEE SMC 2003, Washington D.C. USA.

Watson, M. 1997. Intelligent Java applications. Morgan Kaufmann publishers. San Francisco. USA.

Yassi, A. 1998. Basic Environmental Health. World Health Organization.

\section{AUTHOR BIOGRAPHIES}

JORGE BARDINA, Ph.D., is a Computer Scientist at NASA Ames Research Center, works in the Intelligent Systems Division where he has been leading the Intelligent Launch \& Range Operations project element of NASA's Software, Intelligent Systems, and Modeling element of the Advanced Space Technology Program, in collaboration with Kennedy Space Center and the University of Central Florida. His areas of interests are real-time collaborative environments, web-based and parallel architectures, intelligent systems, modeling, simulation, and engineering technologies. He has authored, co-authored and published over sixty papers and publications. He holds a Ph.D. and M.S. degrees in Mechanical Engineering. and a M.A. degree in Economics from Stanford University. He graduated with top distinction in Civil Engineering of Industries with Mechanical Mention from Universidad Católica de Chile. He has ten years of experience as consultant of major aerospace companies. He is currently the Chairman of the Information Sciences Committee of SAE Aerospace. His email address is: Jorge. E. Bardina@nasa.gov.

RAJKUMAR THIRUMALAINAMBI, Ph. D., is a Computer Scientist at Science Application International Corporation (SAIC), working for NASA Ames Research Center, Moffett Field, California, USA. His research interests are neural networks, fuzzy logic, genetic algorithms, computer simulation and modeling. He is involved in building a virtual test bed for real time simulation of space vehicles using artificial intelligence techniques. His e-mail address is: Rajkumar@mail.arc.nasa.gov. 